\title{
ELEMENTARY FUNCTIONAL EQUATIONS ON PARTIALLY ORDERED LINEAR ALGEBRA
}

\author{
TAEN-YU DAI
}

\begin{abstract}
Suppose that a Dedekind $\sigma$-complete partially ordered linear algebra $A$ admits a function with familiar functional and order properties as possessed by the real-valued exponential, logarithm, root or power function on the real line. Then $A$ can be characterized as an algebra of almost finite extended-real-valued continuous functions defined on some proper topological space.
\end{abstract}

1. Introduction and definitions. This note is motivated by the work in [6] and the study of Cauchy functional equations [1, pp. 37-40], namely, functions having one of the following properties:

$$
\begin{gathered}
f(x+y)=f(x) f(y), \\
f(x y)=f(x)+f(y), \\
f(x y)=f(x) f(y) .
\end{gathered}
$$

On a Dedekind $\sigma$-complete partially ordered linear algebra $A$ (dsc-pola; definition below) with multiplication identity 1 , we shall study those functions having the above property. But such functions always trivially exist; for example, the function $f$ from the dsc-pola $A$ into itself defined by $f(x)=1$, where $x \in A$, has the exponential functional property mentioned above. For this reason, we will study those functions having the additional order property (see theorems in \$2) as possessed by the exponential, logarithm, root or power function on the real line. Using each such function, we are able to characterize its underlying space as a pola of real-valued continuous functions defined on some topological space. We will also discuss the existence of such functions on $A$ and possible general form of these functions.

A dsc-pola, denoted by $A$, is a real linear associative algebra which satisfies the following two conditions: (1) It is partially ordered so that it is a directed partially ordered linear space and $0 \leqslant x y$ whenever $0 \leqslant x, y \in A$. (2) It is Dedekind $\sigma$-complete, i.e., if $x_{n} \in A$ and $x_{1} \geqslant x_{2} \geqslant \cdots \geqslant 0$, then $\inf \left\{x_{n}\right\}$ exists. A dsc-pola has the Archimedean property: if $x, y \in A$ and $\alpha x<y$ for all positive real numbers $\alpha$, then $x \leqslant 0$. Order convergence can be defined as usual. In this paper, we assume $A$ has a multiplicative identity $1 \geqslant 0$. Let $I=\{y \in A: y>1$ and $\left.y^{-1}>0\right\}$ and then define $A_{1}=I-I$. It is shown in [5] that $A_{1}$ is an order-closed

Presented to the Society, January 28, 1977; received by the editors June 28, 1978 and, in revised form, March 5, 1979.

AMS (MOS) subject classifications (1970). Primary 06A70; Secondary 39A40.

Key words and phrases. Dedekind o-complete partially ordered linear algebra, Cauchy functional equations, isotone functions, algebra of almost finite extended-real-valued continuous functions, matrix inequality. 
and order-convex commutative subalgebra of $A$; furthermore, it is an $f$-ring. In fact, $A_{1}$ is algebraically and order isomorphic to an algebra of continuous almostfinite extended-real-valued functions defined on a compact Hausdorff space [9]. We call $A_{1}$ the functional or diagonal part of $A$. For detailed information on $A_{1}$, see [5]; in particular, we refer to the interesting examples on pages 657, 679 and 680 in [5]. It should be noted that the term polac instead of dsc-pola was used in [5].

Lemma 1.1. Suppose a dsc-pola $A$ has an element $x$ and $x<1$. If there exists $0<y \in A$ such that $1 \leqslant x y$ or $1 \leqslant y x$, then $x$ has an inverse and $x^{-1}>1$. In particular, if $x \in A_{1}$, then $x^{-1} \in A_{1}$.

Proof. See Theorems I.6.3 and I.6.10 in [5].

In this paper, there are two frequently used subsets in $A$ which we will denote by $H=\{u \in A: u \geqslant 1\}, K=\{x \in A: x \geqslant 0\}$.

2. Main results. Assuming that a dsc-pola $A$ admits some function $f$ with a familiar functional and order property as possessed by the real-valued exponential, logarithm, root or power function, we show that $A=A_{1}$. The statement of following theorems can be best understood by referring to the Examples 3.1, 3.2, and 3.3; the rest of the examples in that section are intended to show that the order property assumed in each theorem is essential to guarantee $A=A_{1}$.

Our first theorem is related to a function with the exponential property. In this connection, we refer to a problem by Birkhoff; see [4, p. 237].

THeOReM 2.1. Suppose $A$ admits a function $f$ from $A$ into itself such that $f(x+y)$ $=f(x) f(y)$ and $f(x) \geq 1+c x$ for $x, y \in A$, where $c>1$ is a fixed element in $A_{1}$. Then $A=A_{1}$.

Proof. Since $f(0)=f(0)^{2} \geqslant 1$, we have $f(0)=1$. It is clear that for any $x \in A$, $f(x) f(-x)=f(-x) f(x)=f(0)=1$ so that each $f(x)$ has $f(-x)$ as its inverse. From the definition of $A_{1}$, we have $c^{-1} \geqslant 0$. From the definition of $f$, we see easily that $f\left(-c^{-1}\right)>0$. Let $d=c^{-1}$. Then $f(-n d)=f\left(-n c^{-1}\right)=f\left(-c^{-1}\right)^{n}>0$ for all positive integers $n$.

We now show that for any $0<x \in A$, the sequences $\{x f(-n d)\}$ and $\{n f(-n d)\}$ converge to 0 . By the definition of $f$, we see that $f(d)>1+c d=2 \cdot 1$, which implies that $f(n d)=f(d)^{n}>2^{n} \cdot 1$ for all positive integers $n$. Let us multiply $f(-n d)$ on both sides of the last inequality. Using the facts just obtained, we have $1=f(n d) f(-n d) \geqslant 2^{n} f(-n d) \geqslant 0$. It follows that $x / 2^{n}>x f(-n d)>0$ and $\left(n / 2^{n}\right) 1$ $>n f(-n d)>0$ for all positive integers $n$; hence, $\lim x f(-n d)=\lim n f(-n d)=0$.

We next show that for each $0 \leqslant x \in A, f(-x)>0$. From $f(n d-x)>1+$ $c(n d-x) \geqslant n 1-c x$ or $f(-x) f(n d) \geqslant n 1-c x$, we have

$$
f(-x) \geqslant(n 1-c x) f(-n d)=n f(-n d)-c x f(-n d)
$$

for all positive integers $n$. Since $\lim n f(-n d)=\lim \operatorname{cxf}(-n d)=0$, we obtain $f(-x)>$ 0 . This means that $f(x) \geqslant 1$ has a positive inverse $f(-x)$, which by the definition of $A_{1}$ implies that $f(x) \in A_{1}$. Since $A_{1}$ is order-convex and $f(x)>1+c x>x>0$, we get $x \in A_{1}$. Since $A$ is directed, we may conclude that $A=A_{1}$. 
REMARK. It is worth noting that the order property $f(x)>1+c x$ implies that $f$ is an isotone function, namely, for $y, z \in A$ and $y>z, f(y)>f(z)$. This can be proved simply by observing the inequality $f(y) f(-z)=f(y-z)>1+c(y-z)>$ 1 and the fact $f(w)>0$ for any $w \in A$ (because $A$ is directed and $f(-x)>0$ for any $0<x \in A$ ). However, Example 3.4 shows that we do need the stronger condition to get $A=A_{1}$.

Our second theorem is related to an isotone function with the logarithm property.

THEOREM 2.2. Suppose $A$ admits an isotone function $g$ from $H$ into $A$ such that $g(u v)=g(u)+g(v)$ for $u, v \in H$. Suppose also there exists an isotone function $f$ on $A$ such that $f(x+y)=f(x) f(y)$ for $x, y \in A$ and $f(g(u))=u$ for $u \in H$. Then $A=A_{1}$.

Proof. By the definition of $g$, we have $g(1)=0$ so that $f(0)=f(g(1))=1$. For any $0<x \in A$, we show that $f(x) \in A_{1}$. Since $1=f(0)=f(x-x)=f(x) f(-x)$, the inverse of $f(x)$ is $f(-x)$. Since $0 \geqslant-x$ and $f$ is isotone, we see that $1=f(0)>$ $f(-x)$. Let $a=1-f(-x) \geqslant 0$. Then it is clear that $f(-x)=1-a$ and $f(-2 x)=$ $f(-x) f(-x)=(1-a)^{2}$. Using the isotone property of $f$ again, we obtain $f(-x)>$ $f(-2 x)$ or $1-a \geqslant(1-a)^{2}$ so that $a \geqslant a^{2}>0$. It follows trivially that $3(1+a)>$ $(1+a)^{2}>1$. Let us apply $g$ throughout the last inequality. From the definition of $g$, we have $g(3(1+a))>g\left((1+a)^{2}\right) \geqslant 0$ or $g(31)+g(1+a)>2 g(1+a)>0$. Therefore, $g(31) \geqslant g(1+a)>0$; hence, $f(g(31))>f(g(1+a))$ or $31>1+a>$ 0 . This means that $a \in A_{1}$ so that $f(-x)=1-a$ belongs to $A_{1}$. By Lemma 1.1, $f(x) \in A_{1}$.

Let $1<z \in A$. Then $g(z) \geqslant 0$. Applying the result just obtained, we get $z=$ $f(g(z)) \in A_{1}$. Since $A$ is directed, we may conclude that $A=A_{1}$.

Our third theorem is related to an isotone function with the root property.

TheOREM 2.3. Suppose $A$ admits an isotone function $f$ from $K$ into $K$ such that $f(1)=1$ and for each $x \in K, f(x)^{n}=x$, where $n \geqslant 2$ is a fixed integer. Then $A=A_{1}$.

Proof. Choose any $1 \leqslant x \in K$ such that $1<x^{n}-1<x^{n}$. Since $f$ is isotone, we have $1=f(1) \leqslant f\left(x^{n}-1\right) \leqslant f\left(x^{n}\right)$. Let $y=f\left(x^{n}\right)$ and $u=f\left(x^{n}-1\right)$. Hence, $1<$ $u<y$; and furthermore, by the functional property of $f, u^{n}=x^{n}-1=y^{n}-1$ so that

$$
1=y^{n}-u^{n}=y\left(y^{n-1}-u^{n-1}\right)+(y-u) u^{n-1} .
$$

Since $y>u \geqslant 1$, we have $1 \geqslant y\left(y^{n-1}-u^{n-1}\right)>y^{n-1}-u^{n-1}>0$, which means that both $y\left(y^{n-1}-u^{n-1}\right)$ and $y^{n-1}-u^{n-1}$ belong to $A_{1}$. Next, observe that

$$
\begin{aligned}
y^{n-1}-u^{n-1} & =y\left(y^{n-2}-u^{n-2}\right)+(y-u) u^{n-2} \\
& \geqslant(y-u) u^{n-2} \geqslant y-u \geqslant 0 .
\end{aligned}
$$

With $(*)$ and $(* *)$, we see that

$$
\begin{aligned}
y\left(y^{n-1}-u^{n-1}\right)\left(1+u^{n-1}\right) & =y\left(y^{n-1}-u^{n-1}\right)+y\left(y^{n-1}-u^{n-1}\right) u^{n-1} \\
& \geqslant y\left(y^{n-1}-u^{n-1}\right)+(y-u) u^{n-1}=1 .
\end{aligned}
$$


Applying Lemma 1.1 twice, we first get $0 \leqslant\left(y\left(y^{n-1}-u^{n-1}\right)\right)^{-1} \in A_{1}$, then $0<$ $\left(y^{n-1}-u^{n-1}\right)^{-1} \in A_{1}$ (because $\left.\left(y\left(y^{n-1}-u^{n-1}\right)\right)^{-1} y\left(y^{n-1}-u^{n-1}\right)=1\right)$ so that $y \in A_{1}$. Consequently, $x^{n} \in A_{1}$ (recall $x^{n}=y^{n}$ ). Since $1<x<x^{n}$ and $A_{1}$ is order-convex, we have $x \in A_{1}$. Since $A$ is directed, we may conclude that $A=A_{1}$.

REMARK. Using remarks in $\$ 4$, one should be able to see that the $f$ mentioned in Theorem 2.3 is the $n$th root function on $K$. Therefore, $f(x y)=f(x) f(y)$ for $x, y \in$ $K$.

Our last theorem is related to a function with the power function property. We say that $A$ has the decomposition property ((D) property) if for $0<x \in A$, $0<y \in A$ and $0 \leqslant z \leqslant x+y$ there exists $v, w$, in $A$ such that $0<v<x, 0<w$ $<y$ and $z=v+w$. Note that every vector lattice has the (D) property; however, not every ordered vector space for which (D) property holds is a vector lattice. (See $[8$, p. 8].)

THEOREM 2.4. Suppose $A$ admits a function $f$ from $K$ into itself such that $f(x y)=$ $f(x) f(y)$ for $x, y \in K$ and $f(x) \geqslant 1+c(x-1)$, where $c \geqslant \lambda 1, \lambda>1$, is a fixed element in $A_{1}$. Suppose further that $A$ has the (D) property. Then $A=A_{1}$.

Proof. We first show that $f(n 1) \in A_{1}$ for all positive integers $n$. By the definition of $f$, we have $f(1)=f(1)^{2} \geqslant 1$ so that $f(1)=1$. From $1=f((1 / n) 1) f(n 1)=$ $f(n 1) f((1 / n) 1)$ and $f(n 1) \geqslant 1$, it is clear that $f(n 1)$ has a positive inverse $f((1 / n) 1)$, and hence, $f(n 1) \in A_{1}$ for all positive integers $n$.

We next show that there exists a subsequence $\left\{n_{j}\right\}$ such that $\left\{n_{j} f\left(\left(1 / n_{j}\right) 1\right)\right\}$ converges to 0 . Let $j$ be an arbitrary positive integer. Then by the definition of $f$,

$$
f(n 1)=f\left(n^{1 / j} 1\right)^{j} \geqslant\left(1+c\left(n^{1 / j}-1\right) 1\right)^{j} \geqslant\left(\lambda\left(n^{1 / j}-1\right) 1\right)^{j} .
$$

In particular, when $n=n_{j}=j^{j}$,

$$
f\left(n_{j} 1\right) \geqslant(\lambda(j-1) 1)^{j}
$$

It follows that $0 \leqslant f\left(n_{j} 1\right)^{-1} \leqslant(\lambda(j-1) 1)^{-\mathrm{j}}$ or

$$
0 \leqslant f\left(\frac{1}{n_{j}} 1\right) \leqslant \frac{1}{\lambda^{j}(j-1)^{j}} 1,
$$

so that

$$
0 \leqslant n_{j} f\left(\frac{1}{n_{j}} 1\right) \leqslant \frac{n_{j}}{\lambda^{j}(j-1)^{j}}=\frac{1}{\lambda^{j}}\left(\frac{j}{j-1}\right)^{j} .
$$

Since $\lambda>1$ and $\left\{(j /(j-1))^{j}\right\}$ is a bounded sequence, we have $\lim n_{j} f\left(\left(1 / n_{j}\right) 1\right)=$ 0 . In addition, an easy consequence from the last inequality is that for any $x$ in $K$, $\lim n_{j} f\left(\left(1 / n_{j}\right) 1\right) f(x)=0$.

Next, choose any $1 \leqslant x \in A$. Then $f((1 / n) x) \geqslant 1+c((1 / n) x-1)$ for all positive integers $n$. Rearranging terms of the last inequality, we have $n f((1 / n) x)+$ $n(c-1) \geqslant c x \geqslant 0$. Since $A$ has the (D) property, we may write $c x=x_{n}+w_{n}$, where $0 \leqslant x_{n} \leqslant n(c-1)$ and $0 \leqslant w_{n} \leqslant n f((1 / n) x)=n f((1 / n) 1) f(x)$. Let $n=n_{j}$ be as in the above paragraph. Then $\lim n_{j} f\left(\left(1 / n_{j}\right) 1\right) f(x)=0$ so $\lim w_{n_{j}}=0$. Therefore, 
$c x=\lim \left(x_{n_{j}}+w_{n_{j}}\right)=\lim x_{n_{j}}$; hence, $c x \in A_{1}$ (because $x_{n,} \in A_{1}$ and $A_{1}$ is orderclosed). This implies that $x=c^{-1}(c x) \in A_{1}$. Since $A$ is directed, we may conclude that $A=A_{1}$.

3. Examples. The standard real-valued exponential, logarithm, root and power functions on the real line certainly serve as the obvious examples satisfying the conditions of theorems in \$2. Additional examples will be given here to illustrate the basic idea involved in this paper. Examples 3.4 through 3.7 will indicate why these order conditions described in the above theorems are nontrivially chosen to guarantee $A=A_{1}$.

EXAmple 3.1. Let $A=\{x=(\alpha, \beta): \alpha, \beta$ reals $\}$ be the real linear algebra with usual algebraic operations. Partially order $A$ by defining $0<x=(\alpha, \beta)$ iff $0<\alpha$, $0<\beta$. Then $A$ becomes a dsc-pola with $1=(1,1)$ as the multiplicative identity, in fact, $A=A_{1}$. Define $f$ on $A$ as follows: for $x=(\alpha, \beta) \in A, f(x)=\left(3^{\alpha}, 4^{\beta}\right)$. Clearly, $f(x+y)=f(x) f(y)$. Choose $c=(\ln 3, \ln 4)$. Then $c>1, c^{-1}>0$ and it is easy to check that $f(x) \geqslant 1+c x$ for all $x \in A$.

Example 3.2. Let $A$ be as in Example 3.1. Define $g$ on $H$ as follows: for $1<u=(\nu, \delta), g(u)=\left(\log _{3} \delta, \log _{4} \nu\right)$. Then $g$ is an isotone function and $g(u v)=$ $g(u)+g(v)$ for $u, v \in H$.

Next, define $f$ on $A$ by letting $f(x)=\left(4^{\beta}, 3^{\alpha}\right)$, where $x=(\alpha, \beta)$. Then $f$ is an isotone function, $f(x+y)=f(x) f(y)$ and $f(g(u))=u$ for $u \in H$.

Example 3.3. Let $A$ be as in Example 3.1. Define $f$ on $K$ as follows: $f(x)=$ $\left(\alpha^{2}, \beta^{3}\right)$, where $x=(\alpha, \beta)$. Clearly, $f(x y)=f(x) f(y)$ for $x, y \in K$. Choose $c=$ $(2,3)$ and $\lambda=2$. Then it is easy to verify that $c \geqslant \lambda 1$ and $f(x)>1+c(x-1)$ for $x \in K$.

EXAmple 3.4. Let $A=\left\{x=\left[\begin{array}{ll}\alpha & \nu \\ 0 & \beta\end{array}\right]: \alpha, \beta, \nu\right.$ reals $\}$ be the linear algebra with componentwise ordering. Then $A$ becomes a dsc-pola and $A_{1}$ is the set of all diagonal matrices in $A$. Define $f$ on $A$ as follows:

$$
\text { For } x=\left[\begin{array}{ll}
\alpha & \nu \\
0 & \beta
\end{array}\right] \in A, \quad f(x)=\left[\begin{array}{cc}
2^{\alpha} & 0 \\
0 & 3^{\beta}
\end{array}\right],
$$

then $f$ is an isotone function on $A$ which has the exponential functional property $f(x+y)=f(x) f(y)$. However, $A \neq A_{1}$.

Example 3.5. Let $A$ be as in Example 3.4. Define $g$ on $H$ as follows:

$$
\text { For } u=\left[\begin{array}{cc}
\alpha & \nu \\
0 & \beta
\end{array}\right] \in H, \quad g(u)=\left[\begin{array}{cc}
\log _{3} \alpha & 0 \\
0 & \log _{4} \beta
\end{array}\right] \text {. }
$$

Then $g$ is an isotone function on $H$ which has the logarithm functional property $g(u v)=g(u)+g(v)$. Note that $g$ has no inverse function in $A$, but it satisfies an interesting inequality $g(u) \leqslant u-1$ on $H$; yet still $A \neq A_{1}$. This shows the condition $f(g(u))=u$ in Theorem 2.2 is nontrivially chosen to insure $A=A_{1}$. (The reader should observe that the inequality $g(u)<u-1$ is a standard one for the natural logarithm function on the real line.)

EXAMPLE 3.6. Let $A=\left\{x=\left[\begin{array}{cc}\alpha & \beta \\ 0 & \alpha+\beta\end{array}\right]: \alpha, \beta\right.$ reals $\}$ be the algebra with componentwise ordering. Then $A$ becomes a commutative dsc-pola and $A_{1}$ is the set of all 2 by 
2 scalar matrices; hence, $A \neq A_{1}$. Let $z=\left[\begin{array}{ll}0 & 1 \\ 0 & 1\end{array}\right]$. Then $x=\left[\begin{array}{cc}\alpha & \beta \\ 0 & \alpha+\beta\end{array}\right]=\alpha 1+\beta z$. Using this expression, it is not hard to check that each $y \in K$ has a unique positive square root $\sqrt{y}$, i.e. $f(y)=\sqrt{y}$ is well defined on $K$, but $f$ is not an isotone function. This shows that the isotone property of $f$ in Theorem 2.3 cannot be dropped.

Example 3.7. Let $A$ be as in Example 3.6. Define $f$ on $K$ as follows:

$$
\text { For } x=\left[\begin{array}{cc}
\alpha & \beta \\
0 & \alpha+\beta
\end{array}\right] \in K, \quad f(x)=x^{2} .
$$

Since $A$ is a commutative lattice dsc-pola, we have $f(x y)=f(x) f(y)$ on $K$. Although $f$ is isotone on $K$, it does not satisfy the inequality described in Theorem 2.4 .

4. Remarks. Let $A$ be a dsc-pola such that $A=A_{1}$. Then $A$ is an $f$-ring with multiplicative identity 1 as a weak order unit (i.e., if $1 \wedge z=0$, then $z=0$ ). There are various approaches to represent $A$ by an algebra of real-valued continuous functions; see [2], [9]. We will use the results in [9, p. 134, Theorem V.4.1 and p. 139, Theorem V.8.2], which say that $A$ is algebraically and lattice isomorphic to some subalgebra $\hat{A}$ of an algebra $C_{\infty}(Q)$ of continuous, almost finite, extendedreal-valued functions defined on a totally disconnected compact Hausdorff space $Q$. Moreover, $\hat{A}$ is order-convex in $C_{\infty}(Q)$. In the following remarks, we identify $A$ with $\hat{A}$. We first discuss the existence of those functions with the described functional and order property as in $\$ 2$.

REMARK 4.1. If on $H$ we define $g(u)=\ln (u)(\ln (u) \in A$ because $A$ is order-convex) and on $K$ we define $h(x)=x^{1 / n}, p(x)=x^{n}$, where $n>1$ is a fixed integer, then $g, h, p$ are functions having the functional and order property described in Theorems 2.2, 2.3, 2.4 respectively. (Note that $x^{n}>1+n(x-1)$.)

In general, it seems that we cannot determine whether there exists a function on $A$ with the properties stated as in Theorem 2.1. However, if $A=C_{\infty}(Q)$, then for $x \in A, \zeta \in Q, f(x)(\zeta)=\exp (x(\zeta))$, where $\exp (x(\zeta))$ has the usual meaning, is a function from $A$ into itself with the desired functional property and $f(x)>1+x$.

Examples 3.2 through 3.5 and 3.7 show that in general an isotone function $f$ with the property $f(x+y)=f(x) f(y), f(x y)=f(x)+f(y)$ or $f(x y)=f(x) f(y)$ seems to have no solution which can be expressed in general form, but for the type of $f$ as in Theorem 2.1, we have the following result.

REMARK 4.2. Let $f$ be as in Theorem 2.1, $a=\ln (f(1))$ (recall $f(1)>21$ ). Then $f(x)=\exp (a x), x \in A$. (Namely, $f(x)(\zeta)=\exp (a(\zeta) x(\zeta)), \zeta \in Q$.

The proof can be divided into several steps. We sketch them as follows.

STEP 1. If $\left\{x_{n}\right\}$ is a decreasing sequence in $A$. such that $\inf \left\{x_{n}\right\}=0$, then $\inf \left\{f\left(x_{n}\right)\right\}=1$.

By the definition of $f$ and $A_{1}$, we have $f\left(x_{n}\right) \geqslant 1$ and $1-c x_{n}<f\left(-x_{n}\right)<1$. Since $A$ is multiplication continuous [5, p. 652], $\inf \left\{x_{n}\right\}=0$, and $f$ is isotone, we obtain $1=\sup \left\{f\left(-x_{n}\right)\right\}$ so that $\inf \left\{f\left(x_{n}\right)\right\}=1$.

An easy consequence of this is that if $\left\{y_{n}\right\}$ is an increasing sequence in $A$ such that $y=\sup \left\{y_{n}\right\}$, then $f(y)=\sup \left\{f\left(y_{n}\right)\right\}$; in particular, if $y$ is also the pointwise limit of $y_{n}$, then $f(y)$ is the pointwise limit of $f\left(y_{n}\right)$. 
STEP 2. A standard argument shows that for rationals $\alpha, f(\alpha 1)=\exp (\alpha a)$. An easy application of Step 1 yields $f(\nu 1)=\exp (\nu a), \nu$ is any real.

STEP 3. If $0<e^{2}=e<1$, then $f(e)=\exp (a e)$. Note that $e$ is a characteristic function on some open-closed set $E \subset Q$. From $f(e)>1$, we have $1-c e<f(-e)$ < 1. Thus, for $\zeta$ in the complement of $E, f(-e)(\zeta)=1$ or $f(e)(\zeta)=1$. Similarly, $f(1-e)(\zeta)=1$ whenever $\zeta \in E$. From $f(1)=f(e) f(1-e)=\exp (a)$ it follows that for $\zeta \in E, \exp (a(\zeta))=f(1)(\zeta)=f(e)(\zeta) f(1-e)(\zeta)=f(e)(\zeta)$. Therefore, we can write $f(e)(\zeta)=\exp (a(\zeta) e(\zeta)), \zeta \in Q$, or $f(e)=\exp (a e)$.

A simple application of the result in Step 1 leads to $f(\nu e)=\exp (\nu a e)$ for any real $\nu$; hence, for $x=\sum_{i=1}^{n} \nu_{i} e_{i}$, where $0<e_{i}=e_{i}^{2}<1$, we obtain $f(x)=\exp (a x)$.

STEP 4. If $x \in A$ and $0 \leqslant x \leqslant k 1$, then $f(x)=\exp (a x)$.

For such $x$, we know that there exists a sequence $\left\{x_{n}\right\}$ such that $x_{n}$ is a linear combination of characteristic functions $e_{i}$ 's and $\left\{x_{n}\right\}$ converges to $x$ uniformly, i.e., for each $\delta>0$, there exists an integer $n_{0}$ such that for all $n>n_{0},\left|x_{n}-x\right|<\delta 1$ [8, p. 131]. Using the last inequality, it is not hard to see that $f(x)(\zeta)=$ $\lim \left(f\left(x_{n}\right)(\zeta)\right)=\lim \exp \left(x_{n}(\zeta) a(\zeta)\right)=\exp (x(\zeta) a(\zeta))$; hence, $f(x)=\exp (a x)$.

STEP 5. If $y \in A$ and $0 \leqslant y$, then $f(y)=\exp (a y)$.

Let $y_{k}=y \wedge k 1$. Then $0 \leqslant y_{k}<k 1,\left\{y_{k}\right\}$ is an increasing sequence and $\sup \left\{y_{k}\right\}=y$; furthermore, $y$ is also the pointwise limit of $y_{k}$. By the results of Steps 4 and 1 , we have $f(y)(\zeta)=\sup \left\{f\left(y_{k}\right)(\zeta)\right\}=\sup \left\{\exp \left(y_{k}(\zeta) a(\zeta)\right)\right\}=\exp (y(\zeta) a(\zeta))$ or $f(y)=\exp (a y)$.

Since $A$ is directed, it is straightforward to verify that $f(x)=\exp (a x), x \in A$.

ACKNOWLedgement. The main results in $\$ 2$ are based on the conjectures of Professor Ralph DeMarr. The author wishes to thank him for his helpful correspondence.

\section{REFERENCES}

1. J. D. Aczél, Lectures on functional equations and their applications, Academic Press, New York, 1966.

2. S. J. Bernau, Unique representation of archimedean lattice groups and normal archimedean lattice rings, Proc. London Math. Soc. 15 (1965), 599-631.

3. G. Birkhoff, Lattice theory, Amer. Math. Soc. Colloq. Publ., vol. 25, 3rd ed., Amer. Math. Soc., Providence, R.I., 1967. MR 37 \#2638.

4. P. F. Conrad and J. E. Diem, The ring of polar preserving edomorphisms of an Abelian lattice-ordered group, Illinois J. Math. 15 (1971), 222-240.

5. T. Y. Dai, On some special classes of partially ordered linear algebras, J. Math. Anal. Appl. 40 (1972), 649-682.

6. T. Y. Dai and R. DeMarr, Isotone functions on partially ordered linear algebras with a multiplicative diagonal map, Proc. Amer. Math. Soc. 65 (1977), 11-15.

7. R. DeMarr, A class of partially ordered linear algebras, Proc. Amer. Math. Soc. 39 (1973), 255-260.

8. A. L. Peressini, Ordered topological vector spaces, Harper and Row, New York, 1967.

9. B. Z. Vulikh, Introduction to the theory of partially ordered spaces, Wolters-Noordhoff, Groningen, 1967.

Department of Mathematics, York College, Jamaica, New York 11451 